\title{
Flow over a wall-mounted finite-length square cylinder
}

\author{
J. Chen \\ Mechanical, Automotive and Materials Engineering \\ University of Windsor \\ Windsor, Canada \\ chen112p@gmail.com
}

\author{
R. Balachandar \\ Mechanical, Automotive and Materials Engineering \\ University of Windsor \\ Windsor, Canada \\ rambala@uwindsor.ca
}

\author{
R.M. Barron \\ Mechanical, Automotive and Materials Engineering \\ University of Windsor \\ Windsor, Canada
}

\begin{abstract}
A conventional way of studying the flow characteristics of flow over a bluff body is by extracting the velocity distribution in horizontal planes crossing the bluff body near the bed, mid-height and close to the free-end location. The intention of selecting the planes at these levels is to examine the horseshoe vortex and the bed effect, flow separation at the mid-height, and the downwash flow from the free-end. In some cases, where a relatively short cylinder is studied along with a taller cylinder, the mid-height level of each may present very different flow structures. It is proposed in this study that a better way of selecting the planes of interest could be based on the pressure distribution imposed on the front surface of the bluff body by the approaching flow. This paper uses a sample numerical study of wall-mounted finitelength square cylinder with three aspect ratios of 1, 2 and 4, immersed in the air flow with a thin boundary layer. The horizontal planes based on the front face pressure distribution are selected and examined to show the advantage of the proposed method.
\end{abstract}

Keywords: Bluff body; flow separation; Detached-eddysimulation

\section{INTRODUCTION}

A wall-mounted finite-length square cylinder immersed in a flow field has many engineering applications in the real world, such as flow over low-rise buildings, skyscraper stacks, chimney stacks, etc. The aspect ratio $(A R=h / d$, where $h$ and $d$ are the height and width of the object, respectively) plays an important role in establishing the flow features around the body (Kawamura et al. [1]). As AR increases, the shear layer in the overhead region induced by the top edge becomes more energetic and then interacts with the base vortices. This phenomenon slowly diminishes when AR approaches a critical value.
When an oncoming flow with a boundary layer impinges on the front surface of a square cross-section cylinder, a stagnation point (or zone) is induced on the front side of the cylinder surface (Fig. 1). The location of the stagnation point $\left(\mathrm{h}_{\text {stg }}\right)$ is defined by the maximum value or the middle point of the near-constant region on the pressure distribution of the front surface. The flow below the stagnation streamline rolls up into the horseshoe vortex (HSV), while the flow above it separates from the top edge of the body. The separated flow forms the wake region as indicated in the schematic.

When discussing the wake structures behind the cylinder of different AR, researchers tend to present results in different horizontal planes. These planes usually include the one at middepth $(50 \% \mathrm{~h})$, one near the wall $(25 \% \mathrm{~h})$ and one near the free-end of the cylinder $(75 \% \mathrm{~h})$. However, the induced flow structures are not simply decided by the cylinder's height. When comparing flow past cylinders of different height, it is quite possible that the flow structures are different at a certain value of the cylinder height to width ratio. For instance, the mid-height level of a short cylinder may be heavily influenced

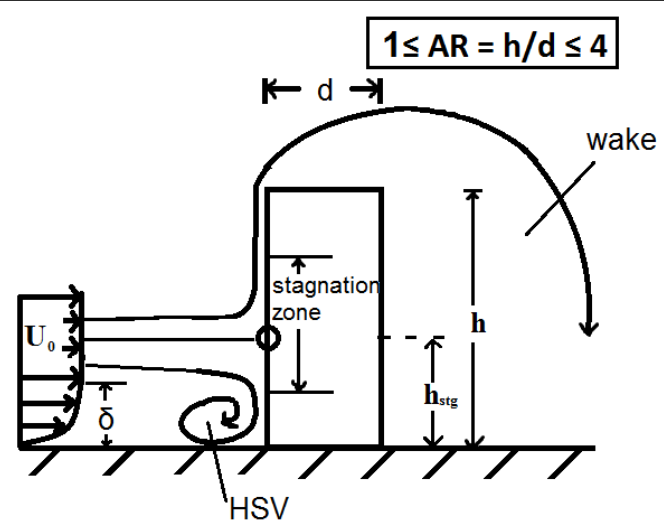

Fig. 1 Central plane schematic of flow over a wall-mounted finitelength square cylinder 
by the bed-effect, but the mid-height level of a taller object may not be influenced either by the distance to the wall or to the top edge of the body. A new scheme based on the pressure distribution on the object's front surface which could be potentially used in the analysis of the flow over a bluff body problem is introduced in this paper.

\section{COMPUTATIONAL DOMAIN}

The schematic of the flow field is shown in Fig. 2. A wallmounted square cylinder with the width (d) of $12.7 \times 10^{-3} \mathrm{~m}$ is immersed in an air flow. The air flow is at a Reynolds number of 12,000 (based on freestream velocity and cylinder width), with the free stream velocity of $15 \mathrm{~m} / \mathrm{s}$ and dynamic viscosity of $1.855 \times 10^{-5} \mathrm{~Pa}$-s. Three aspect ratios $(\mathrm{AR}=1,2$ and 4$)$ of the square cylinder are studied.

The origin of the coordinate system is located at the center of the square at the bed level. The $\mathrm{x}-, \mathrm{y}$ - and $\mathrm{z}$ - coordinate directions correspond to the streamwise, spanwise and normal directions, respectively. The two side-walls and the ceiling are slip-walls, and the bottom bed and the cylinder faces are noslip walls. The square cylinder is placed $6 \mathrm{~d}$ downstream of the inlet, $40 \mathrm{~d}$ upstream of the pressure outlet, $7 \mathrm{~d}$ under the ceiling (9d and $10 \mathrm{~d}$ for $A R=2$ and $A R=1$, respectively), and $15 \mathrm{~d}$ away to each side-wall. The side length of the computational domain is guided by the work of Nasif et al. [2], which showed the need for a distance of $15 \mathrm{~d}$ between each side-wall and the cylinder to maintain a small enough blockage ratio. This length is larger than that used by Saeedi and Wang [3] in their direct numerical simulation (DNS) of a similar flow field.

In the experimental study by El Hassan et al. [4], the cylinder was placed at the location along the bottom wall where the boundary layer thickness was $9.1 \times 10^{-3} \mathrm{~m}(72 \% \mathrm{~d})$ in the absence of the cylinder. To properly benchmark the numerical setup with the experiment, the inlet condition is set to reproduce the velocity profile at the same location in the absence of the cylinder. Based on a grid independency study, the proper grid was chosen to simulate the flow field using Detached Eddy Simulation. The results were validated using the experimental results of El Hassan et al. [4] in several horizontal and vertical planes through the wake.

\section{RESULTS AND DISCUSSION}

Fig. 3 shows the pressure distribution on the front surface of the cylinder along the central plane $(y / d=0)$. Two points of

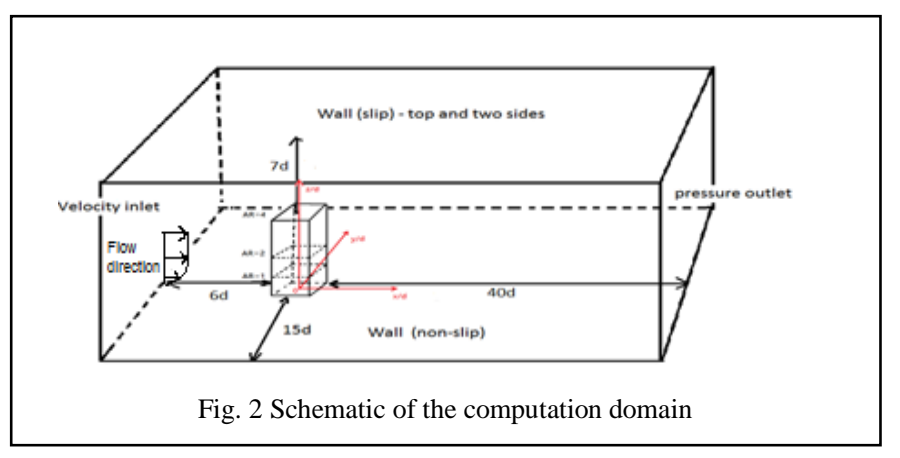

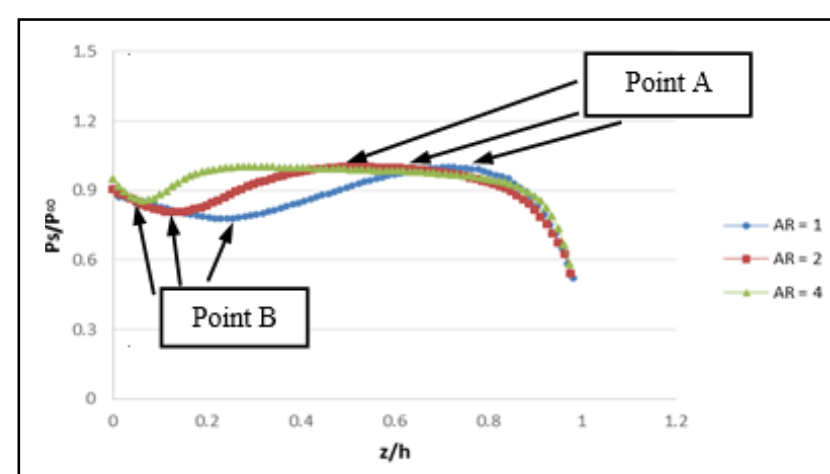

Fig. 3 Pressure coefficient distribution on the front surface of the cylinder at central plane $\mathrm{y} / \mathrm{d}=0$

interest are labeled as Point $\mathrm{A}$ and Point $\mathrm{B}$, which are the maximum pressure (or maximum pressure zone) on the surface and local minimum pressure, respectively. Point A characterizes the stagnation point in each case. Only in the case of $\mathrm{AR}=1$, a distinctive maxima is found on the cylinder's front surface at $\mathrm{z} / \mathrm{d}=0.727$, while the cylinders $\mathrm{AR}$ $=2$ and 4 have a near constant pressure region. In the cases of $\mathrm{AR}=2$ and 4, Point $\mathrm{A}$ is marked at the center of the constant pressure region, which are $\mathrm{z} / \mathrm{d}=1.2$ and 2 , respectively.

As seen in Fig. 3, the constant pressure region increases in size as the cylinder AR increases. Since the free-end of the cylinder moves further away from the bed, there is more space in the middle section that is not influenced by either end. The stagnation point's location with respect to the cylinder width increases as the AR increases, and the stagnation point's location with respect to the cylinder's height decreases as the cylinder's AR increases.

Point $\mathrm{B}$ describes the local minimum pressure on the front surface and lies closer to the bed region. The location of Point $\mathrm{B}$ with respect to the cylinder's width are relatively close for all three ARs. Therefore, Point B can be understood as a critical point induced by the bed effect, namely the horseshoe vortex. The core of HSV (where the highest vorticity is found) is roughly half of the distance between Point B and the bed. If the HSV is seen as a symmetric structure about its core, the incoming flow at the level of Point $\mathrm{B}$ in each case is at the upper boundary of the HSV.

By extracting the velocity field on the horizontal planes passing through Point A and Point B, flow structures for each AR are expected to share similar features. Therefore, the comparison between different ARs would be more meaningful than only considering the comparison at a certain cylinder height to width ratio, especially when one cylinder is significantly shorter than the other.

Figs. 4 (a), (b), and (c) show the time-averaged streamwise velocity on a horizontal plane passing through Point A for AR $=1,2$, and 4, respectively. The flow separates at the sides of the cylinder and accelerates in the downstream direction. The flow with high velocity slowly reduces as it moves downstream. Even though the size of the wake structure and 


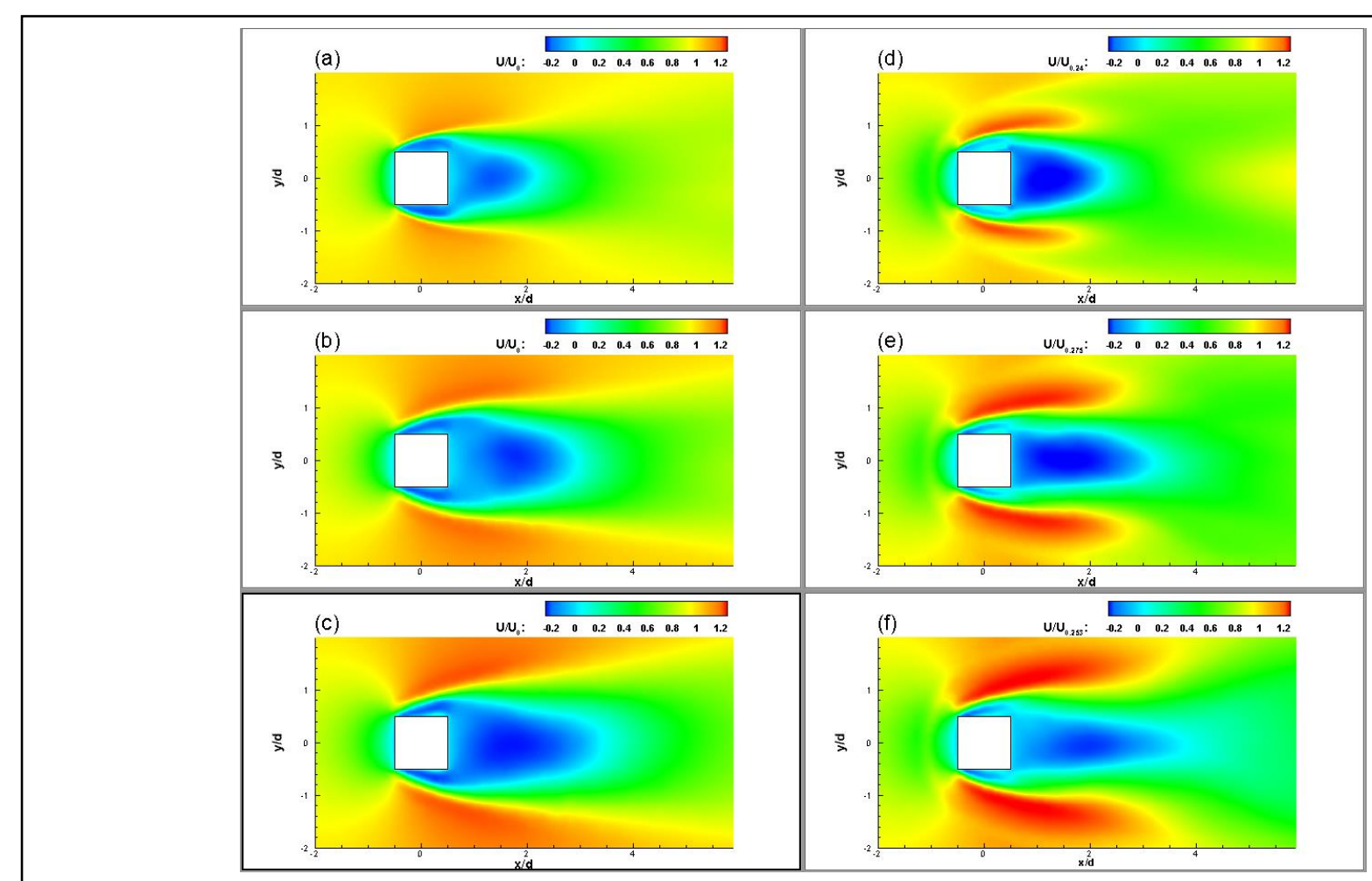

Fig. 4 Horizontal planes shaded by the time-averaged streamwise velocity at the location of Point A: (a), (b) and (e) for the cylinder AR $=1,2$, and 4 respectively; and Point B: (d), (e), and (f) for the cylinder AR =1,2, and 4 respectively.

the value of the streamwise velocity are different, the contour of the flow structures shows many similarities.

Figs. 4 (d), (e), and (f) show the time-averaged streamwise velocity on a horizontal plane passing through Point B for AR $=1,2$, and 4 , respectively. The planes at Point B show many different features than the planes at the stagnation point (Point A), while the planes at Point B share a lot of similarities among themselves. Two zones (red and blue regions), which represent the separating shear layer (outer) and the collar vortex (inner) (Nasif et al. [2]), lie on each side of the cylinder. Although the characteristics of the flow structures are different due to the increase of AR, the flow structures presented are similar.

\section{CONCLUSION}

The comparison made by selecting the planes based on the pressure distribution show similar structures in the flow field at all aspect ratios. The pressure distribution on the front face is clearly a function of the aspect ratio. The choice of the horizontal planes based in the maximum and minimum pressure values enables a direct comparison at different value of AR. Further analysis of the use of the front face pressure distribution is necessary to understand the characteristics of the flow past bluff objects.

\section{REFERENCES}

[1] Kawamura, T., Hiwada, M., Hibino, T., Mabuchi, I. \& Kumada, M. "Flow around a finite circular cylinder on a flat plate," Bull. JSME 27, 2142-2150, 1984

[2] Nasif, G., Balachandar, R., Barron, R.M., "Characteristics of flow structures in the wake of a bed-mounted bluff body in shallow open channel," Journal of Fluids Engineering, Vol 137 / 101207-1, 2015

[3] Saeedi, M., Wang, BC., "Large-eddy simulation of turbulent flow around a finite-height wall-mounted square cylinder within a thin boundary layer," Flow Turbulence Combust 97, 513-538, 2016

[4] El Hassan, M., Bourgeois, J., Martinuzzi, R., "Boundary layer effect on the vortex shedding of wall-mounted rectangular cylinder," Exp. Fluids, $56: 33,2015$ 